\title{
ARQUEOLOGIA DO TRABALHO IMATERIAL: uma aplicação bibliométrica à análise de dissertações e teses ${ }^{\mathrm{i}}$.
}

\author{
ARCHEOLOGY OF THE IMMATERIAL WORK: the application of the \\ bibliometric analysis on dissertations and thesis
}

\author{
Nair Yumiko Kobashi - nykobash@usp.br \\ (PPGCI/ECA-USP ) \\ Raimundo Nonato Macedo dos Santos - raimundo@cin.ufsc.br \\ (PGCIN/UFSC)
}

\section{Resumo}

Busca de alternativas teóricas e metodológicas para mapear a pesquisa científica brasileira. Apresenta-se como hipótese de trabalho a idéia de que os repositórios de dissertações e teses podem ser fontes confiáveis para conhecer a ciência produzida no país. Por meio de procedimentos que associam os Estudos Sociais da Ciência, a Organização e Representação do Conhecimento e Métodos bibliométricos avançados, podem ser vislumbradas novas abordagens sobre a institucionalização social e cognitiva da pesquisa científica no Brasil. A visualização gráfica dessas informações, por meio de cartografias dinâmicas, é útil por sua funcionalidade para oferecer visão global de conjuntos de informações e evidenciar relações entre elas. Da mesma forma, as visualizações configuram-se como possíveis interfaces para recuperação de informação.

Palavras-chave: Institucionalização da pesquisa científica. Organização do conhecimento. Cartografia temática. Indicadores bibliométricos. Dissertações e teses.

\section{INTRODUÇÃO}

Os trabalhos de Whitley (1974), realizados no campo da Sociologia da Ciência, são referências teóricas úteis para abordar a institucionalização da pesquisa científica. Segundo o modelo por ele proposto, a pesquisa científica pode ser analisada segundo duas categorias: a institucionalização cognitiva e a institucionalização social.

A institucionalização cognitiva diz respeito aos aspectos epistemológicos, teóricos e metodológicos das disciplinas científicas. São seus objetos os graus de consenso sobre os conceitos da área, a legitimidade e pertinência dos problemas propostos ao campo, o grau de aceitabilidade das soluções encontradas e, finalmente, o grau de adequação dos métodos, técnicas e instrumentos de observação dos fenômenos.

A institucionalização social, por sua vez, refere-se às estruturas formais que demarcam os membros de uma comunidade científica. São analisados, nesta dimensão, os graus de organização de áreas do conhecimento, sua integração às estruturas de legitimação (universidades, institutos de pesquisa, instâncias de avaliação, sociedades científicas, etc) e as condições de acesso a programas de fomento.

Alguns comentários preliminares são necessários para contextualizar o estudo da institucionalização cognitiva e social da pesquisa no campo da Ciência da Informação, 
como também para justificar a abordagem aqui proposta:

1) os produtos da pesquisa científica, vistos na ótica da economia informacional, por serem expressões do trabalho imaterial (HARDT e NEGRI, 2002), podem ser descritos e analisados para revelar aspectos da institucionalização da ciência.

2) trabalhos de escavação da produção intelectual, como o aqui proposto, não são novos. De fato, inspira-se em trabalho seminal de Foucault - "A arqueologia do saber". Seu comentário sobre o porquê da escolha desse título tem servido de base para muitos trabalhos sobre os processos de construção do conhecimento:

Inicialmente, empreguei essa palavra de maneira um pouco cega, para designar uma forma de análise que não seria efetivamente uma história (no sentido em que se relata, por exemplo, a história das invenções e das idéias), e que tampouco seria uma epistemologia, ou seja, uma análise interna da estrutura de uma ciência. Trata-se de uma coisa diferente, e então eu a chamei de "arqueologia"; depois, retrospectivamente, pareceu-me que o acaso não tinha me guiado muito mal: afinal, essa palavra "arqueologia", ao preço de uma aproximação que me será perdoada, eu espero, pode querer dizer: descrição do arquivo. Por arquivo, entendo o conjunto de discursos efetivamente pronunciados; e esse conjunto é considerado não somente como um conjunto de acontecimentos que teriam ocorrido uma vez por todas e que permaneceriam em suspenso, nos limbos ou purgatórios da história, mas também como um conjunto que continua a funcionar, a se transformar através da história, possibilitando o surgimento de outros discursos (FOUCAULT, 2000, p. 145).

Este texto fundamenta-se epistemologicamente nas formulações de Hardt e Negri (2002) e Foucault (2000), acima enunciadas. Pretende-se, desse modo, empreender uma arqueologia do trabalho imaterial, sob a ótica da Ciência da Informação. Para isso, serão exploradas as bases de dados referenciais de dissertações e teses produzidas em programas de pós-graduação do país.

Não se reivindica originalidade neste trabalho. A afirmação de Granger sobre o processo de construção do conhecimento: a descontinuidade radical dos paradigmas é um mito, porque os modelos científicos sempre se estabelecem "a partir de um momento anterior do pensamento" (GRANGER, 1989, p.25), balizam os limites deste trabalho e as vozes que reverberam como panos de fundo. Portanto, esta pesquisa se apóia nos estudos anteriores já realizados por pesquisadores da área da Ciência da Informação e campos conexos.

Explorar as bases de dados de dissertações e teses produzidas no país, descrevê-las e produzir indicadores tem o sentido, portanto, de rememorar e reavaliar a atividade científica desenvolvida na universidade. $O$ projeto proposto requer abordagem interdisciplinar que entrecruza três saberes: os Estudos Sociais da Ciência, a Organização e Representação do Conhecimento e os Métodos Bibliométricos avançados.

São dados empíricos da pesquisa, as dissertações e teses produzidas nos programas de pós-graduação do país, que serão analisadas por métodos bibliométricos. 
Os estudos bibliométricos, na visão tradicional, fundam-se na descrição e na quantificação. $\mathrm{Na}$ abordagem aqui proposta combinam-se estudos quantitativos e qualitativos, explorando-se a oposição entre qualidade e quantidade, com base em Granger (1989), que afirma que essa relação pode ser explicada como uma oposição ontológica.

Como lembra Granger (1989), remonta a Descartes o reconhecimento do duplo sentido do quantitativo: a enumeração e a medida. Nessa dicotomia, a quantidade se associa ao número inteiro natural e tem com o objeto apenas uma relação extrínseca. No caso da medida, ao contrário, opera-se com o tratamento quantificado de propriedades que podem ser submetidas a operações de disjunção e de reunião. A quantificação é, portanto, uma operação mais complexa do que a enumeração.

Ainda segundo Granger (1989), o conhecimento qualitativo, pode também ter um duplo sentido: referir-se à idéia de diferença ou à idéia de forma. A diferença é obtida por meio de operações ativas de classificação, portanto, de disjunção e de reunião, tal como na quantificação.

A dicotomia quantidade/qualidade se expressa nas formas de abordar os objetos: descrever, compreender, explicar (GRANGER, 1989). O processo de descrição consiste primeiramente em escolher as dimensões dos dados empíricos que serão, em seguida, divididos em elementos discretos e enumeráveis. A descrição gera, portanto, um conhecimento morfológico que evidencia um todo e as suas partes. Um conhecimento qualitativo, por sua vez, procura compreender e explicar. Porém seria presunçoso imaginar que se possa compreender sem primeiramente descrever de forma adequada $o$ objeto que se analisa.

$\mathrm{Na}$ Ciência da Informação, são controvertidas as relações entre a quantificação e o conhecimento qualitativo. Procura-se, nesta pesquisa, explorar a idéia de que o conhecimento qualitativo pode ser objetivado por relações quantificadas, provindas da aplicação de técnicas bibliométricas. Afirma-se, portanto, que um conhecimento qualitativo não elimina a quantidade. Ao contrário, procura-se tomar a medida como meio para compreender e explicar, de modo a quebrar a clivagem entre o modo quantitativo e o modo qualitativo de analisar objetos.

Em estudos bibliométricos é necessário, primeiramente, descrever os elementos constitutivos das bases de dados (os dados de input), de modo a estabelecer conjuntos. Esses conjuntos se aproximam por "semelhanças de família", no sentido proposto por Wittgenstein (1996). O conceito de "semelhança de família" dá conta de objetos que se relacionam, não por uma constelação de caracteres que os transformam em classes rigorosas, em que os objetos da classe compartilham um núcleo totalmente invariante. São, na realidade, tipos de agrupamentos que apresentam alguma inexatidão e fluidez, como o são os descritores das linguagens documentárias. No entanto, para garantir a confiabilidade dos resultados da análise é necessário que sejam expostos os parâmetros de operação de constituição dessas categorias operacionais. Rejeitam-se, portanto, as operações tradicionais das técnicas bibliométricas que enumeram sem analisar o objeto para determinar os critérios de composição das famílias de dados. 


\section{A APLICAÇÃo BIBLIOMÉTRICA À ANÁLISE DE DISSERTAÇÕES E TESES.}

\subsection{Breve caracterização da bibliometria}

A bibliometria é uma metodologia de recenseamento das atividades científicas e correlatas, por meio de análise de dados que apresentem as mesmas particularidades. Por meio dessa metodologia, pode-se, por exemplo, identificar a quantidade de trabalhos sobre um determinado assunto; publicados em uma data precisa; publicados por um autor ou por uma instituição ou difundidos por um periódico científico, o grau de desenvolvimento de P\&D e de inovação, entre outros. Por meios bibliométricos pode-se, por exemplo, computar dados para comparar e confrontar os elementos presentes em referências bibliográficas de documentos representativos das publicações.

Os estudos bibliométricos objetivam conhecer como se comporta uma propriedade relativamente a outra já conhecida. Dessa forma, no caso das publicações científicas, pode-se pretender conhecer todos os autores que trabalharam em determinado assunto; os periódicos que publicaram sobre esse assunto; os autores considerados referência no assunto; os autores desse assunto que publicaram em determinado periódico e assim por diante e cruzar essas propriedades.

A classificação do resultado da contagem dos elementos, por ordem de freqüência de aparição decrescente, produziu as primeiras distribuições estatísticas em bibliometria. Os estudos sobre tais distribuições constituem, assim, os primórdios das aplicações estatísticas à Produção Científica (POLANCO, 2003).

Atualmente, há consenso entre os estudiosos que atuam na área, de que o campo da bibliometria, como um todo, inclui os aspectos quantitativos e os modelos da comunicação científica e do armazenamento, disseminação e recuperação da informação científica. Esse conceito de bibliometria é, nesse enfoque, mais amplo do que as definições usuais do termo e objetiva incorporar todas as orientações correntes, como também suas aplicações à política científica e à recuperação da informação (WORMELL, 1998).

Os métodos bibliométricos vêm sendo aplicados não somente aos estudos cientométricos (métrica dos aspectos da ciência, enquanto disciplina ou atividade econômica) (MACIAS-CHAPULA, 1998), e à avaliação da pesquisa em ciência e tecnologia (C\&T), mas também à análise de seus aspectos sociais e econômicos. Com efeito, o mapeamento e a avaliação da ciência são questões consideradas cruciais, na sociedade contemporânea, principalmente a partir de sua caracterização como fator de produção.

A avaliação da pesquisa científica pode ser qualitativa ou quantitativa. A avaliação feita por pares é de natureza qualitativa, sendo utilizada, em geral, para julgar propostas de pesquisa, avaliar grupos de pesquisa e artigos científicos, entre outros aspectos. Os indicadores bibliométricos, por sua vez, representam o aspecto quantitativo do processo avaliativo. Deve-se observar, no entanto, que os aspectos quantitativos também estão presentes na avaliação por pares, já que, nessas avaliações, também são contados o número de publicações e as atividades acadêmicas próprias das áreas institucionalizadas 
(VAN RAAN, 2003).

Os indicadores bibliométricos vêm ganhando importância crescente como instrumentos para análise da atividade científica e das suas relações com o desenvolvimento econômico e social. Sua construção tem sido incentivada pelos órgãos de fomento à pesquisa como meio para se obter uma visão acurada da produção de ciência, de modo a subsidiar a política científica e avaliar seus resultados.

Os indicadores são, desse modo, parâmetros empregados como medidas indiretas da atividade da pesquisa científica e contribuem para a compreensão dos objetivos da pesquisa, das estruturas da comunidade científica, do seu impacto social, político e econômico (SPINAK, 1998; TRZESNIAK, 1998; OKUBO,1997). Quando empregados na análise da produção científica, os indicadores bibliométricos podem ser divididos em indicadores de produção, indicadores de citação e indicadores de ligação (MACIASCHAPULA, 1998; SPINAK, 1998; OKUBO, 1997; NARIN et alii, 1994; COURTIAL, 1990; CALLON et alii, 1993).

Os Indicadores de produção científica são construídos pela contagem do número de publicações por tipo de documento (livros, artigos, publicações científicas, relatórios etc.), por instituição, área de conhecimento, país, etc.

Os indicadores de citação, por sua vez, são construídos pela contagem do número de citações recebidas por uma publicação de artigo de periódico. É o meio mais reconhecido de atribuir crédito ao autor.

Já os indicadores de ligação são construídos pela co-ocorrência de autoria, citações e palavras, sendo aplicados na elaboração de mapas de estruturas de conhecimento e de redes de relacionamento entre pesquisadores, instituições e países. Emprega técnicas de análise estatística de agrupamentos.

\subsection{Exemplo de mapeamento bibliométrico}

Em complemento às aplicações para obtenção de indicadores da ciência, tecnologia e inovação, os estudos recentes da área concentram-se em técnicas de mapeamento bibliométrico para monitorar o desenvolvimento científico (básico ou aplicado), uma vez que eles são considerados instrumentos úteis para identificar padrões na estrutura dos campos científicos, identificar processos de disseminação do conhecimento e visualizar as dinâmicas do desenvolvimento científico, tecnológico e de sua efetiva adoção na produção de bens e serviços.

Os mapas gerados a partir desses dados e métodos são representações da produção científica de áreas. Semelhante produção pode ser expressa por meio de conceitos produzidos e utilizados pela própria área. Tem-se, portanto, neste caso, garantia literária na geração das representações cartográficas da institucionalização cognitiva de um dado campo do conhecimento.

A visualização da informação por meio de mapas baseia-se nos estudos sobre a percepção, que mostram que o ser humano tem primeiro uma percepção global de uma cena antes de atentar para os detalhes (TUFTE, 1983). Tais estudos abriram caminhos 
para explorar as características da percepção global e, conseqüentemente, para aplicá-las aos sistemas de informação, particularmente nos aspectos relacionados à recuperação de informação, tais como:

- Exploração rápida de conjuntos de informações desconhecidas;

- Evidenciação de relações e estruturas nas informações;

- Fornecimento de alternativas de acesso a informações pertinentes;

- Classificação interativa de informação.

Para demonstrar como as cartografias podem ser utilizadas para descobrir padrões na estrutura dos campos científicos, identificar processos de disseminação do conhecimento e visualizar as dinâmicas do desenvolvimento científicos, são apresentadas, a seguir, cartografias, construídas com base em métodos de análise multivariável $^{\text {ii. }}$

Essas análises consistem na comparação de todos os pares possíveis dos descritores que geram, neste caso particular, dois tipos de gráficos, para uma mesma observação: a) gráficos de classificação hierárquica - dendograma - e, b) classificação não hierárquica - multidimensional.

Os mapas abaixo apresentados (Figura 1 e Figura 2), visualizam os descritores das 97 teses e dissertações defendidas no Programa de pós-graduação do Instituto de Pesquisas Nucleares de São Paulo, dentro da categoria S63 da base de dados INIS. A categoria S63 compreende temas relativos à "Radiation - thermal pollutant effects - biological, Material and Living organisms".

$\mathrm{Na}$ Figura 1, a visualização da dinâmica da aplicação de processo científico de "radiação gama à base de cobalto 60 " sobre os mais diversos tipos de matérias e organismos vivos evidencia, de forma objetiva, as relações e as estruturas que foram, ao longo dos últimos 30 anos de pesquisa no IPEN, hierarquicamente, estabelecidas entre as temáticas de estudo.

Na Figura 2, em que se estuda a mesma categoria S63, o objetivo foi o de representar em espaço reduzido, os "clusters" que se formaram entre as temáticas estudadas, a partir dos processos de radiação gama à base do cobalto 60 , bem como as relações que se estabelecerem entre os diversos clusters. 
Figura 1: Classificação hierárquica de descritores - DENDOGRAMA

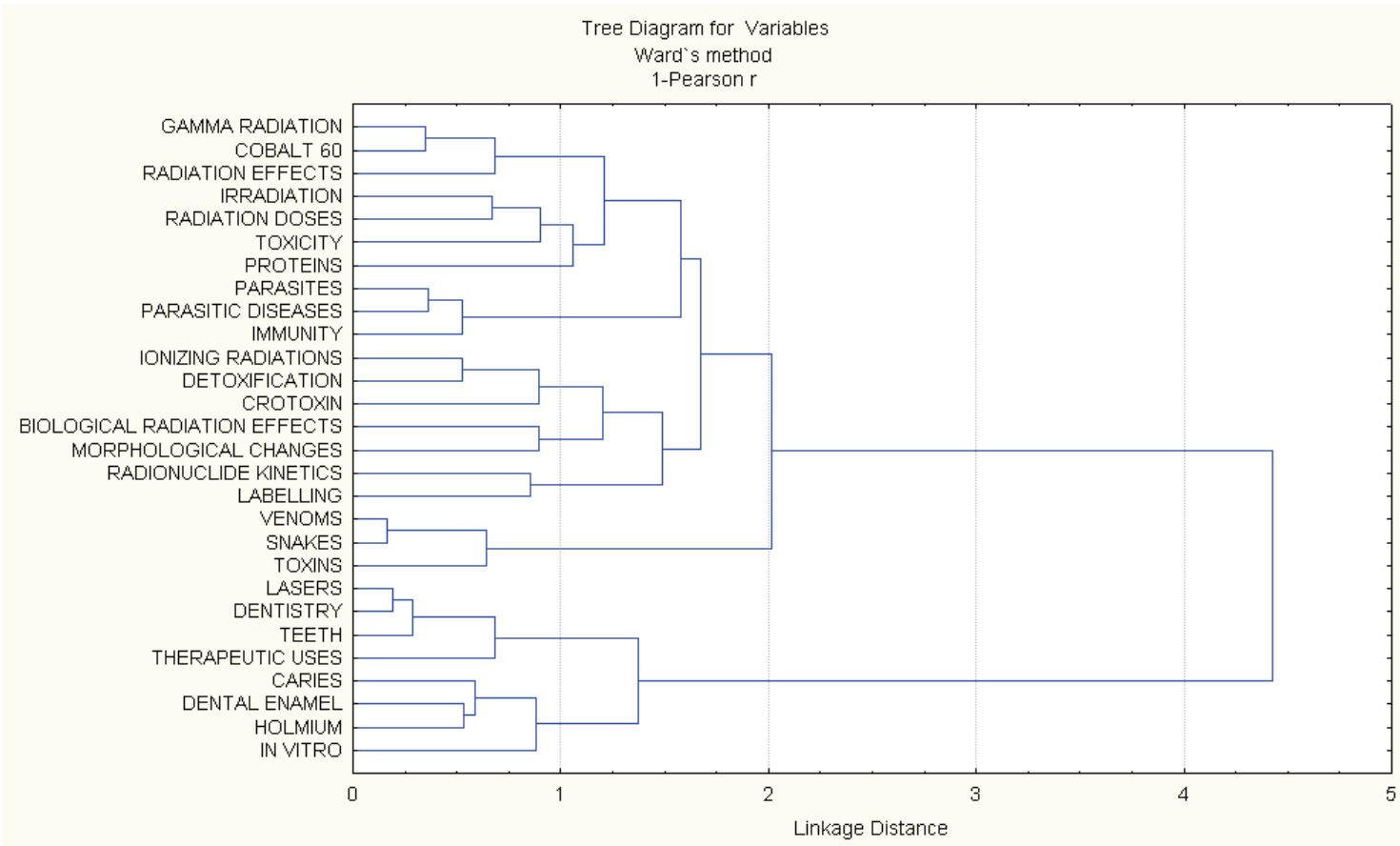

Figura 2: Classificação, em espaço reduzido, não hierárquica de descritores - ESCALA MULTIDIMENSIONAL (MSD)

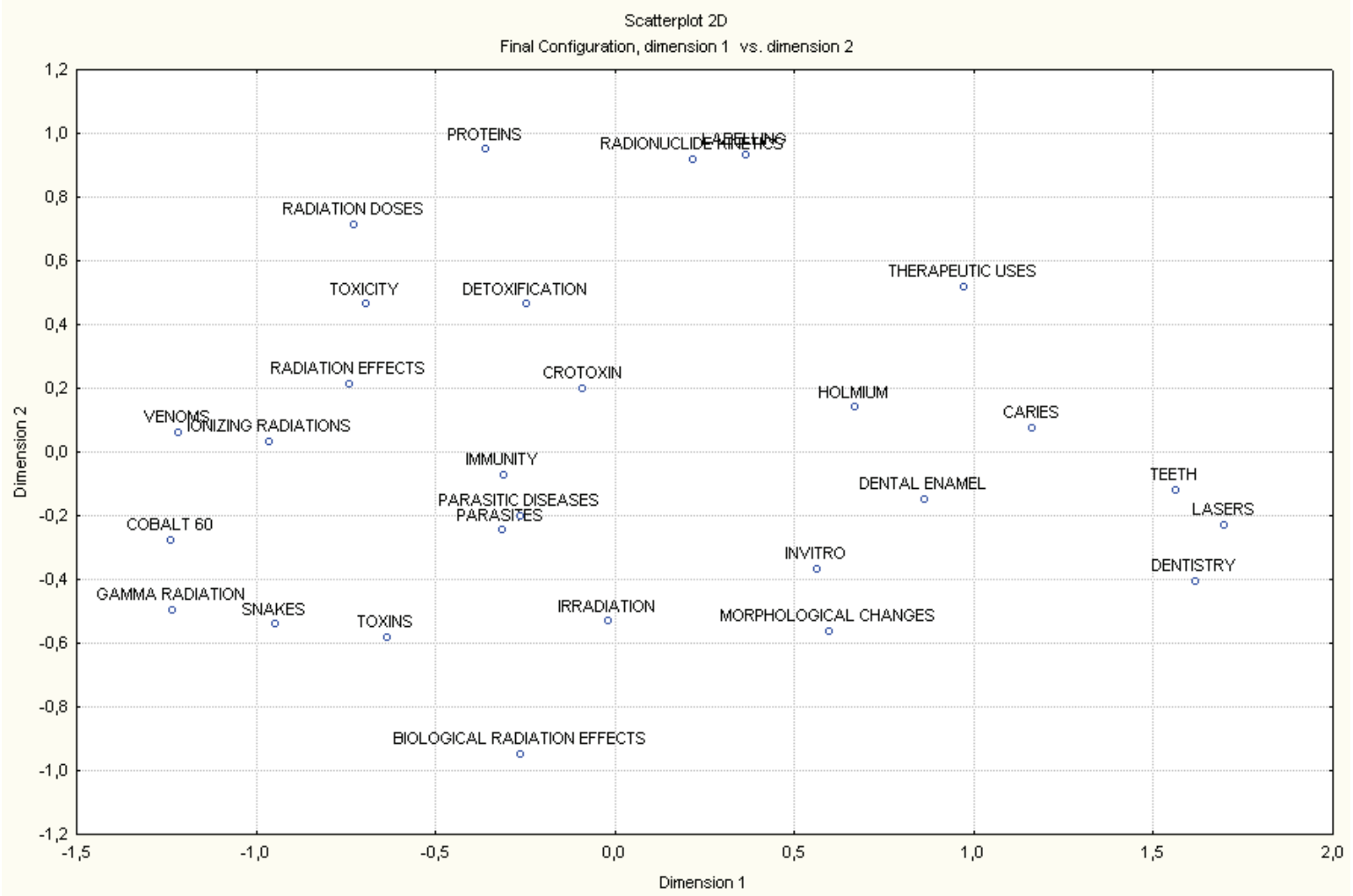




\section{CONSIDERAÇÕES FINAIS}

Foram apresentados, neste trabalho, exemplos do que pode ser obtido com a exploração das bases de dados por meio de técnicas bibliométricas avançadas. O estudo combina abordagem quantitativa e qualitativa, com o intuito de descrever, conhecer e explicar fenômenos. Procurou-se demonstrar que os dispositivos de memória institucionalizados, dentre eles as Bases de Dados referenciais, são inscrições que, se adequadamente analisadas, podem gerar representações da cultura científica do país. No entanto, tais artefatos têm tido seu uso limitado à localização de informação ou como instrumentos de apoio à gestão de acervos. Analisar tais inscrições, descobrir relações entre os dados e apresentá-los sob a forma de cartografias estáticas ou dinâmicas, é um modo de resgatar a produção de conhecimento que se faz no país. Esse resgate pode se configurar, além disso, como insumo para promover a produção de novos conhecimentos e para orientar as políticas de pesquisa. Para serem eficazes, no entanto, tais análises devem ser objeto de produção contínua.

Deve-se chamar a atenção também para o fato de que as especificidades de cada país quanto ao estágio de institucionalização da ciência apontam para a necessidade de redefinir pressupostos antes de empreender a tarefa de produzir indicadores. É preciso, ademais, conhecer em profundidade as características e hábitos de cada setor para elaborar hipóteses de trabalho pertinentes e utilizar metodologias de coleta e análise que produzam resultados confiáveis. No caso brasileiro, o desafio é maior dada a precariedade ou mesmo ausência de fontes de informação para empreender estudos bibliométricos.

Outro aspecto a ser enfatizado refere-se aos próprios métodos bibliométricos. Estes, se aplicados de forma cega, podem gerar resultados pouco confiáveis. A crítica constante dos procedimentos é, portanto fundamental. Os problemas que surgem requerem respostas que, muitas vezes, são encontradas em outras especialidades. Se os estudos sociais da ciência são fundamentais para interpretar os dados, os métodos da Organização da Informação são imprescindíveis para reorganizar os dados em categorias funcionais adequadas para a análise das tendências de pesquisa.

O mapeamento das tendências globais de institucionalização cognitiva e social da pesquisa científica, por meio de técnicas bibliométricas avançadas, parece, portanto, ser cada vez mais imperioso. Somente com dados objetivados podem ser justificadas e sustentadas as decisões sobre as políticas de pesquisa. Sua ausência, não raro, resulta em decisões frágeis porque baseadas apenas em impressões ou em cristalização de posições que não encontram eco na dinâmica da criação científica. Tais decisões acabam por engessar um campo científico em lugar de promover seu desenvolvimento e consolidação. É sabido que esses resultados dependem, muitas vezes da abertura de fronteiras, promovida por meio do diálogo intra ou interdisciplinar.

\section{REFERÊNCIAS}

CALLON, M.; COURTIAL, J-P.; PENAN, H. La scientométrie. Paris, Presses Universitaires de France, collection “Que sais-je?", v. 2727, 1993, 126p.

COURTIAL, J.P. Introduction à la scientométrie: de la bibliométrie à la veille 
technologique. Paris: Anthropos, 1990.

FOUCAULT, M. Arqueologia das ciências e história dos sistemas de pensamento. Rio de Janeiro: Forense, 2000.

GRANGER, G-G. Por um conhecimento filosófico. Campinas: Papirus, 1989.

HARDT, M.; NEGRI, A. Império. Rio de Janeiro: Record, 2002.

MACIAS-CHAPULA, C.A. O papel da informetria e da cienciometria e sua perspectiva nacional e internacional. Ciência da Informação, Brasília, v. 27, n. 2, p. 134-140, maio/ago. 1998.

NARIN, F.; OLIVASTRO, D.; STEVENS, K. S. Bibliometric theory, practive and problem. Evaluation Review, v. 18, n. 1, 1994.

OKUBO, Y. Bibliometric indicators and analysis of research systems: methods and examples. . Paris, OECD, 1997, 69 p. (STI Working Papers, 1997/1).

POLANCO, X. Aux sources de la scientométrie: Dossier Solaris, No.2, Bibliométrie, Scientométrie, Infométrie. Available from: $<$ http://www.info.unicaen.fr/bnum/jelec/Solaris/d02/2polanco1.html $>$ Acesso em: 16/01/2003.

SANTOS, R.N.M. Desenvolvimento de indicadores da produção científica da Ciência da Informação no Brasil. Projeto de pesquisa desenvolvido na PUC-Campinas, 2005.

SANTOS, R.N.M.; KOBASHI, N,Y. Aspectos metodológicos da produção de indicadores em ciência e tecnologia. Apresentado no VI CINFORM. Salvador, 17 de junho de 2005.

SPINAK, E. Indicadores cienciométricos. Ciência da Informação, Brasília, v. 27, n. 2, p. 141-148, 1998.

TRZESNIAK, P. Indicadores quantitativos: reflexões que antecedem seu estabelecimento. Ciência da Informação, Brasília, v. 27, n. 2, p. 159-164, maio/ago., 1998.

TUFTE, E.R. The virtual display of quantitative information. Cheshire, Connecticut: Graphics Press, 1983.

VAN RAAN, A.F.J. The use of bibliometric analysis in research performance assessment and monitoring of interdisciplinary scientific developments. Pre-print, 2003.

WHITLEY,R. Cognitive and social institutionalization of scientific specialities and research areas. In: WHITLEY,R. (ed) Social processes of scientific development. London: Routledge and Kegan, 1974, p. 69-95

WITTGENSTEIN, L. Investigações filosóficas. Petrópolis: Vozes, 1996.

WORMELL, I. Informetria: explorando bases de dados como instrumentos de análise.

Ciência da Informação, v.27, n.2, p. 210-216, 1998.

\begin{abstract}
Search for theoretical and methodological alternatives for reliably mapping scientific research. In order to face such challenge, we present the hypothesis that dissertations and theses files may be reliable sources to know the country's produced science. By means of an interdisciplinary approach which associates the Social Science Studies, the Knowledge Organization and Representation and advanced Bibliometric Methods, new approaches can be aroused on new forms of social and cognitive science institutionalization in Brazil. Their graphic visualization, by means of dynamic cartography, is useful for its functionality by offering a global vision of information sets as well as evidencing relationships amongst them. Likewise, it is configured as interface
\end{abstract}


for information retrieval systems.

KEYWORDS: Scientific research institutionalization. Knowledge organization. Bibliometric studies. Science mapping. Dissertations.

Originais recebidos em: 08/02/2008

Texto aprovado em: 13/03/2008

i Versão preliminar deste texto foi apresentada no VII Enancib, realizado em 2006, em Marília (SP).

ii Conjunto de métodos de análise numérica, que trata em bloco planilhas de dados (em geral matriz retangular), em que cada elemento da amostra é definido por várias variáveis 